\title{
PARECERISTAS DO VOLUME 26
}

Alberto Cupani - Departamento de Filosofia - UFSC - SC

Alberto Villani - Instituto de Física - USP - SP

Aldoir Rigoni - Faculdade de Física - PUCRS - RS

Alice Assis - Faculdade de Engenharia de Guaratinguetá - UNESP - SP

André Koch Torres de Assis - Instituto de Física - UNICAMP - SP

André Maurício Brinatti - Departamento de Física - UEPG - PR

Andréia Guerra - Colégio Pedro II - RJ

Arden Zylbersztajn - Departamento de Física - UFSC - SC

Carlos Eduardo Laburú - Departamento de Física - UEL - PR

Cassio Stein Moura - Departamento de Física - PUCRS - RS

Cibelle Celestino Silva - Instituto de Física - USP - São Carlos - SP

Cristiane Tavolaro - Departamento de Física - PUC - SP

Daniela Franco Carvalho Jacobucci - Instituto de Biologia - Universidade Federal de Uberlândia - MG

Deise Miranda Vianna - Instituto de Física - UFRJ - RJ

Délcio Basso - Instituto de Física - PUC - RS

Eduardo Koji Takahashi - Universidade Federal de Uberlândia - MG

Elder Sales Teixeira - Departamento de Física - Universidade Estadual de Feira de Santana - BA

Eliezer Batista - Departamento de Matemática - UFSC - SC 
Eloneid Felipe Nobre - Departamento de Física - Universidade Federal do Ceará $-\mathrm{CE}$

Erika Zimmermann - Faculdade de Educação -UnB - DF

Ervino Carlos Ziemath - Instituto de Geociências e Ciências Exatas - UNESP Rio Claro - SP

Fernanda Ostermann - Instituto de Física - UFRGS - RS

Fernando Lang da Silveira - Instituto de Física - UFRGS - RS

Fernando Siqueira da Silva - Bolsista PIBIC - CNPq - Universidade de Caxias do Sul - Instituto Elizabeth Randon - Caxias do Sul - RS

Flávia Rezende - Laboratório de Tecnologias Cognitivas - NUTES - UFRJ - RJ

Flávio Renato R. de Lima - Departamento de Física - UFSC - SC

Francisco Caruso - CBPF - Instituto de Física Armando Dias Tavares - UERJ Rio de Janeiro - RJ

Francisco Catelli - Departamento de Física e Química - Universidade de Caxias do Sul - RS

Frederico F. de Souza Cruz - Departamento de Física - UFSC - SC

Hector Riveros - Instituto de Física - UNAM - México

Helena Libardi - Departamento de Física e Química - Universidade de Caxias do Sul - RS

Isabel Martins - Nutes - UFRJ - RJ

Ivanilda Higa - Departamento de Teoria e Prática de Ensino - UFPR - PR

Joan Portolés - Depto. de Didáctica de les Ciéncies - Universitat de Valencia Espanha

João Batista Canalle - Instituto de Física - UERJ - RJ

João Batista S. Harres - Instituto de Física - PUC - RS 
Joaquim N. B. de Moraes - Departamento de Física - UFSC - SC

Jorge Roberto Pimentel - Departamento de Física - UNESP - SP

Jorge Valadares - Universidade Aberta - Lisboa - Portugal

José Cláudio Reis - Colégio Pedro II - RJ

José de Pinho Alves Neto - Instituto Federal de Educação, Ciência e Tecnologia de Santa Catarina - SC

José Maria Filardo Bassalo - Departamento de Física -UFPa

Juliana Mesquita Hidalgo Ferreira - Departamento de Física Teórica e Experimental - UFRN - RN

Kepler de Souza Oliveira Filho - Instituto de Física - UFRGS - RS

Marcelo Gomes Germano - Departamento de Física - Universidade Estadual da Paraíba - PB

Marcelo Magalhães Fares Saba - Instituto Nacional de Pesquisas Espaciais - SP

Marco Aurélio Alvarenga Monteiro - Departamento de Física - Instituto Tecnológico de Aeronáutica - SP

Marco Braga - CEFET - RJ

Marcos Daniel Longhini - Faculdade de Educação - Universidade Federal de Uberlândia - MG

Marcus Benghi Pinto - Departamento de Física - UFSC - SC

Maria Conceição Barbosa Lima - Departamento de Física - UERJ - RJ

Maria de Fátima Oliveira Saraiva - Instituto de Física - UFRGS - RS

Maria de los Ángeles Fanaro - Universidad Nacional del Centro de la Provincia de Buenos Aires - Buenos Aires - Argentina

Maria José P. M. de Almeida - Faculdade de Educação - UNICAMP - SP 
Marisa Franzoni - Unisal - Campinas - SP

Maurício Pietrocola de Oliveira - Faculdade de Educação - USP - SP

Nelson Canzian - Departamento de Física - UFSC - SC

Nelson Vani Leister -Instituto de Astronomia, Geofísica e Ciências Atmosféricas - USP - SP

Nilva Sales - Universidade Paulista - SP

Odilon Giovannini - Centro de Ciências Exatas e Tecnologia - Universidade de Caxias do Sul - RS

Orlando Aguiar - Faculdade de Educação - UFMG - MG

Osmar Henrique Moura da Silva - Departamento de Física -UEL - PR

Paulo Miranda - Instituto de Física - UFBA - BA

Paulo Rômulo de Oliveira Frota - Universidade do Extremo Sul Catarinense - SC

Rejane Ribeiro Teixeira - Instituto de Física - UFRGS - RS

Ricardo Francke Sandoval - Instituto de Física - UFRGS - RS

Ricardo Gauche - Instituto de Química - UnB - Brasília DF

Ricardo Roberto Plaza Teixeira - Centro Federal de Educação Tecnológica de São Paulo - SP

Rodrigo Drumond Vieira - Doutorando em Educação - Faculdade de Educação $\mathrm{UFMG}-\mathrm{MG}$

Sayonara Cabral da Costa - Departamento de Física - PUC - RS

Sérgio de Mello Arruda - Departamento de Física - UEL - PR

Sônia Krapas Teixeira - Departamento de Física - UFF - RJ

Susana Maria Coelho - Departamento de Física - PUC - RS

Tatiana da Silva - Departamento de Física - UFSC - SC 
Thirza Pavan Sorpreso - Faculdade Comunitária de Santa Bárbara - SP

Ubiratan D'Ambrósio - Instituto de Matemática - UNICAMP - SP

Wagner Duarte José - Departamento de Ciências Exatas e Tecnológicas Universidade de Santa Cruz - BA 